\title{
El protagonismo de las fundaciones americanas en la institucionalización de la sociología (1945-1960)
}

\author{
Josep Picó \\ Universitat de València. Departament de Sociologia \\ Josep.Pico@uv.es
}

\section{Resumen}

Este artículo expone el protagonismo que desempeñaron las fundaciones Rockefeller, Carnegie y Ford en la institucionalización de la sociología después de la Segunda Guerra Mundial. Estas instituciones han tenido grandes cantidades de dinero a su disposición y la mayor parte del trabajo empírico en ciencias sociales sólo se puede llevar a cabo si hay dinero disponible para cubrir sus gastos. Pero, ¿quiénes se beneficiaron de estas ayudas en Europa y Estados Unidos?

El dinero público y privado ha desempeñado un papel significativo y cada vez mayor en el desarrollo de la sociología, pero cabe también preguntarse cómo ha afectado a la investigación el origen de este dinero. La Ford, por ejemplo, tuvo un papel importante en la introducción del behaviorismo aplicado al análisis político, y algunos sociólogos piensan que las fundaciones han estado vinculadas al poder y al capitalismo, lo cual ha condicionado la orientación de las ciencias sociales. Pero otros consideran que esto no es motivo suficiente para explicar el impacto del dinero sobre los métodos sociológicos, y creen que esta explicación requiere también otros factores. ¿Podemos ofrecer ya una lectura alternativa del comportamiento de las fundaciones en relación con las ciencias sociales?

Palabras clave: fundaciones, Rockefeller, investigación empírica, survey, S. Stouffer, ciencias del comportamiento, R. Dahl, T. Parsons.

\section{Abstract. The role of American Foundations in Sociology Institutionalization (1945-1960)}

This paper states the role of foundations Rockefeller, Carnegie and Ford in the institutionalization of sociology after de Second World War. The leading foundations have had enormous sums of money at their disposal and much empirical work can only be carried out if funding is avalaible to cover its expenses. But, which were the beneficiaries in Europe and United States?

Private and public funding has played a significant and increasing role for sociology, but the question of how research is affected by the patterns of available funding is, also, a significant one. Ford has played and important role in introducing the behaviorism in the political analysis, and some sociologist have tended to assume that foundations are obviously associated with power and capitalisme, but others consider that it cannot provide a sufficient explanation on the impact of funding on sociological methods; other factors are also logically required. Has time come to offer an alternative account of the reasons for foundation behavior in relation to the social sciences?

Key words: foundations, Rockefeller, empirical research, survey, Stouffer, behavioral sciences, R. Dahl, T. Parsons. 


\section{Sumario}

1. Antecedentes 4. Las fundaciones en el ámbito

2. La política de las fundaciones en USA y Europa

3. Funcionamiento y orientación científica de la política cultural americana 5. Los fondos estatales sustituyen a las fundaciones

6. La crítica a las fundaciones

\section{Antecedentes}

Aunque en algunos casos excepcionales la investigación en el campo de la sociología ha sido apoyada por mecenas privados, como lo fue El campesino polaco en Europa y América (W.I. Thomas y F. Znanieki, 1918-20) ${ }^{1}$, sin embargo podemos decir que la mayoría de los recursos destinados a esta área del conocimiento después de la Segunda Guerra Mundial han venido de las grandes fundaciones particulares o del Estado. Existe ya abundante literatura sobre el tema, de la que queremos destacar en lengua inglesa los trabajos de R.F. Arnove, G. Alchon, M. Bulmer, D. Fisher o J. Platt ${ }^{2}$, y en lengua francesa, B. Mazon y Revel-Watchtel ${ }^{3}$. Cómo se ha ejercido este apoyo en el caso de la sociología, en qué medida afectó al desarrollo de la disciplina y cuáles han sido las críticas que los propios científicos han dirigido a esta tarea es lo que vamos a describir en este artículo, aunque lo primero que deberíamos destacar es que de las grandes cantidades de dinero que tanto las fundaciones como el Estado han dedicado a las ciencias sociales en el período estudiado (1945-1960) la sociología ha sido una de las áreas menos beneficiada.

En el ámbito de las fundaciones americanas la primogenitura y el protagonismo lo tuvo siempre la Fundación Rockefeller ${ }^{4}$, aunque la Carnegie le acompañó en esta tarea cuyo peso asumió más tarde la Ford, y tanto el interés y el respaldo de estas fundaciones como el del Estado comenzó mucho antes de la Segunda Guerra Mundial.

John D. Rockefeller decidió, en 1910, crear una gran institución filantrópica que tuviese una misión mundial cuya lógica de acción debía ser "promover el bienestar de la humanidad», y para ello era necesario no solamente ocu-

1. Thomas, W.I.; Znaniecki, F. (1918-20). The Polish peasant in Europe and America. Boston: Badger.

2. ARNOVE, R.F. (ed.) (1982). Philanthropy and cultural imperialism. Bloomington, Indiana: University Press; AlCHON, G. (1985). The invisible hand of planning. Princeton University Press. Una bibliografía más detallada se encuentra también en PLATT, J. (1996). A history of sociological research methods in America 1920-1960. Cambridge: Cambridge University Press.

3. MAZON, B. (1988). Aux origines de l'École des Hautes Études en Science Sociales. Le rôle du mécénat américain (1920-1960). París: Les Éditions du Cerf; ReVEL, J.; WaCHTEL, N. (1996). Une école pour les sciences sociales. París: Les Éditions du Cerf.

4. FISHER, D. (1983). "The role of philantropic foundation in the reproduction and production of hegemony: Rockefeller foundations and the social sciences». Sociology, 17, p. 206-233; Collier, P.; Horowitz, D. (1976). Une dynastie américaine, les Rockefeller. París: Seuil. 
parse de la salud de las personas humanas, sino también del equilibrio social y de su preservación en todo el planeta. Según sus propias palabras:

[...] las ciencias que se dedican a la descripción de los fenómenos sociales están poco desarrolladas y carecen de recursos y estímulo institucional. Por tanto el desarrollo de las ciencias sociales necesita ante todo quince o veinte instituciones de investigación bien establecidas y eficaces porque en el momento actual no hay ninguna 5 .

A esta tarea se va a dedicar la Fundación Laura Spelman Rockefeller, que crea, en 1923, el Social Science Research Council (SSRC), de cuyos fondos pronto se van a beneficiar las principales universidades americanas (Harvard, Columbia, Chicago, Yale), así como algunas de las europeas más importantes ${ }^{6}$.

La Fundación Rockefeller proporcionará recursos para el desarrollo de las ciencias sociales, según una pauta científica basada en la observación de los hechos: esta pauta rompe con la tradición europea de trabajo científico impregnada de filosofía y reflexión especulativa. El director de la Fundación — cuenta la historiadora B. Mazon- tenía la ambición de llevar las ciencias sociales a un nivel de cientificidad parecido al de las ciencias físicas y naturales y financiar solamente proyectos concretos y actuales. Para ello era necesario también formar una generación nueva de investigadores, por lo cual se planificó un programa muy amplio de becas de estudio que se aplicó a partir de $1924^{7}$.

Uno de los ejemplos más ilustrativos de la filosofía política que ejerció la Fundación Rockefeller en este periodo fue su proyección en Francia. Para apoyar las actividades de investigación en una sociedad de grandes recursos donde las ciencias sociales permanecían en un estado muy primario la fundación pidió dos proyectos, uno al economista Charles Rist, profesor de la Facultad de Derecho y subgobernador del Banco de Francia, y otro al antropólogo Marcel Mauss. Al primero la Fundación le concedió una credibilidad inmediata (350.000 dólares durante siete años), porque entendió que hablaban el mismo lenguaje, mientras que Mauss, que no entendía por qué la Fundación daba muchos millones a los programas de investigación en Inglaterra y Alemania y no a Francia, vio rechazadas sus pretensiones.

En su proyecto Mauss había propuesto la construcción de un nuevo edificio para el conjunto de las ciencias humanas y quería reorganizar la investigación alrededor de los institutos de la Universidad de París. Sugirió también la puesta en marcha de una encuesta sobre la ciudad de París como las que la Fundación Rockefeller había encargado para Chicago, esta investigación, según Mauss, «se convertiría en un monumento de la sociología y popularizaría inme-

5. FosDICK, R.B. (1952). The story of the Rockefeller Foundation. Nueva York: Harper and Brothers; MAZON, B. (1985). «La fondation Rockefeller et les sciences sociales en France, 1925-40». Rev. Franc. de Sociologie, XXVI, p. $311-342$.

6. Bulmer, M. (1982). «Support for sociology in the 1920s: The Laura Spelman Rockefeller Memorial». The American Sociologist, V, p. 185.

7. MAZON, B. (1985), op. cit., p. 318. 
diatamente, en Francia y en París, el instituto que tratamos de fundar, impulsando otras investigaciones y la formación de estudiosos que tanta falta hacen en este campo de las ciencias» ${ }^{8}$.

Pero la Rockefeller esperaba de él un programa sobre problemas sociales concretos, con una definición clara de los métodos que tuviesen como finalidad la mejora del control social, y se encontró con un proyecto muy general, de concepción liberal y pluridisciplinaria. La fundación desconfió de un socialista, sobrino de Durkheim, que no había hecho nunca estudios aplicados sobre el terreno.

Sin embargo, tampoco dudó en apoyar el Centro de Documentación Social de la Escuela Normal Superior, sostenido débilmente por el banquero Albert Khan, que, bajo la dirección del durkhemiano C. Bouglé, reuniría a estudiosos como G. Friedmann, R. Aron y más tarde J. Stoetzel, quienes, según la Fundación, deberían dedicar su tiempo a la investigación de la sociología inductiva aplicada sobre todo a la organización de la industria francesa, lo cual contribuyó a la orientación de la sociología francesa en los años treinta.

Pero entre las dos guerras no sólo la Fundación Rockefeller, sino también la Guggenheim, la Carnegie, la American Council y otras concedieron becas y ayudas para que los americanos estudiasen fuera, patrocinasen conferencias y periódicos, fundasen librerías o financiasen bibliotecas y profesores visitantes. Las fundaciones sirvieron como un canal a través del cual se establecieron relaciones personales e institucionales entre ambas partes del océano.

La Rockefeller concedió ayudas a Oxford para la renovación de su biblioteca Bodleian, a la London School of Economics, a las universidades de Berlín, Rotterdam y a muchas otras, de tal manera que en 1934 había dado ya dieciocho millones de dólares para el desarrollo de las ciencias sociales y quince para intercambios académicos 9 .

En el ámbito gubernamental conviene recordar ${ }^{10}$ que en esa época el enfoque «constructivista» de la política científica americana comenzó con la administración Hoower y continuó a un ritmo más acelerado con su sucesor F. Roosevelt. H. Hoower tenía un concepto ingenieril del Estado y entendió el mandato de presidente como una gran tarea de ingeniería social, de tal manera que creó el President's Research Committee on Social Trends (1930-32) para vincular la ciencia social con la política social, tarea que fue apoyada por la Fundación Rockefeller a través del Social Science Research Committee. Esta demanda coincidió con la crisis de la economía americana, y los políticos trataron de responder de manera pragmática a la cuestión "conocimiento para qué?» que había lanzado R. Lynd en 1939. H. Laswell fue uno de los primeros que trató de responder la postura desafiante de Lynd ${ }^{11}$ confirmando la

8. Mauss, M. (1985). "Les sciences sociales à París vues par M. Mauss». Rev. Française de Sociologie, vol. XXVI, 2, p. 343-352.

9. Pells, R. (1997). Not like us. Nueva York: Basic Books.

10. Horowitz, I.L. (1970). «Social Science and public policy». Transactions of the sixth World Congress of Sociology. Milan, p. 63.

11. LyND, R. (1939). Knowledge for what? Nueva Jersey: Princeton University Press. 
necesidad del conocimiento para aumentar la eficacia y operatividad de la política federal en áreas como la salud, el bienestar y tantas otras que, como había demostrado la tradición inglesa, servían para reformular políticas sociales ${ }^{12}$.

En este periodo de 1930 a 1945 el incremento de las organizaciones dedicadas a las ciencias sociales contribuyó de manera decisiva a construir la imágen ideológica del científico social profesional. Aumentaron los niveles de precisión metodológica y comenzaron a desaparecer los prejuicios entre políticos y científicos. Un colaborador destacado de la comisión Hoower durante los años de la Depresión fue el sociólogo William Ogburn, cuyos indicadores sociales fueron una pieza fundamental para el National Resources Planning Board, y también participó durante la posguerra en las negociaciones sobre el apoyo del gobierno a las ciencias sociales ${ }^{13}$.

\section{La política de las fundaciones en USA y Europa}

Pero la relación de las fundaciones con la sociología, su impulso y orientación tuvo mucho mayor impacto durante la guerra y sobre todo después de ella. Las instituciones más beneficiadas en Estados Unidos fueron sus universidades, que se convertirían en grandes centros de investigación y puntos de referencia para muchos sociólogos europeos. Columbia, Harvard y Chicago se llevaron gran parte de las ayudas a la financiación por parte de estas instituciones "privadas» en el periodo de posguerra. Harvard pasó de ser un colegio de élite a ser una universidad ambiciosa que competía por tener los mejores estudiantes en todas las disciplinas académicas.

Según Turner ${ }^{14}$, el argumento que esgrimieron los científicos universitarios para captar estos fondos fue que el sistema de financiación de las fundaciones privadas era más adecuado para los grandes institutos de investigación que el del Estado, porque preservaba la creatividad de los investigadores y su libertad individual. Sin embargo esta ventaja se pervirtió poco después debido, en primer lugar, a que el incremento de los intermediarios y gestores de las fundaciones dio pie para que ocuparan estos puestos muchos científicos frustrados o incluso profesores convertidos en consejeros y técnicos, y también porque más tarde, con la muerte de los gestores que habían estado en contacto con el fundador, este tipo de trabajo se convirtió en una carrera con sus imperativos de competitividad, jerarquía y todos los problemas que ello comporta. Ahora bien, si una fundación como la Carnegie quería prestigiarse tenía que escoger colaboradores universitarios, y como consecuencia de esto muy a menudo se intercambiaba personal entre la universidad y las fundaciones: B. Ruml, por

12. CaRdwell, D.S.L. (1957). The organization of science in England: A Retrospect. Londres: Heinemann.

13. KlaUsner, S.Z.; Lidz, V.M. (eds.) (1986). The nazionalization of the social sciences. Pensylvania: University of Pensylvania Press.

14. Turner, S.P.; Turner, J.A. (1990). The impossible Science. An Institutional analysis of american sociology. Londres: Sage. 
ejemplo, que fue el director de la fundación Rockefeller durante muchos años, llegó a ser decano en la universidad de Chicago; R. Lynd tuvo cargos en los dos tipos de instituciones; S. Stouffer fue muchos años consultor de instituciones filantrópicas, y $\mathrm{B}$. Berelson empezó como académico y más tarde fue reclutado por la fundación Ford, como también ocurrió con E. Shils. De esta manera sucedía que los mayores beneficiarios de las fundaciones eran casi siempre las grandes universidades.

En Europa tres grandes países gozaron del favor de las fundaciones: Francia, Inglaterra y Alemania. En Francia los primeros contactos se iniciaron entre el director general de enseñanza superior del Ministerio de Educación, Pierre Auger, y el secretario de Estado americano John Marshall, y fueron propiciados por C. Levi-Strauss, entonces agregado cultural de la embajada francesa, con la finalidad de perfilar el modo de contribuir la Fundación Rockefeller al desarrollo de las ciencias humanas. Se trataba de crear la VI Sección dentro de la École des Hautes Études para sacar la economía y las ciencias sociales de la Facultad de Derecho, con un primer núcleo de personas formado por especialistas en economía e historia de las ciencias y las técnicas. En la primera lista de nombres que Auger remitió a la fundación figuraban L. Febvre, A. Koyré, F. Braudel, Mozaré y G. LeBrass entre otros, que coinciden con el comité de dirección de los Annales, tal como se había configurado después de la guerra. Se trataba de formar la nueva institución con trabajadores atraídos por el estudio de "cuestiones económicas y sociales». Su director será L. Febvre, como secretario se escogió a F. Braudel y su presupuesto de cuatro millones de francos será financiado en una cuarta parte por la Rockefeller ${ }^{15}$.

Pero otras muchas instituciones francesas también se beneficiaron de la Rockefeller como la Fundación de Ciencias Políticas, el Instituto Científico de Economía Política, la Escuela Politécnica e incluso el Centre National de la Recherche Scientifique (CNRS).

Aún así hasta 1948 no se creó un centro de investigaciónes sociológicas propio de la VI Sección. La razón fue que ya existía el CES (Centro de Estudios Sociológicos) creado por G. Gurvitch en 1946, que mantenía muy buenas relaciones de colaboración con el CNRS, de tal manera que las primeras encuestas que puso en marcha la VI Sección se llevaron a cabo conjuntamente con el CES y fueron dirigidas por G. Le Bras y G. Friedmann.

Unos años después, cuando Braudel y Heller pidieron ayuda para el edificio de lo que más tarde sería la Maison des Sciences de l'Homme, la Rockefeller se opuso categóricamente porque no se interesaba ya por el desarrollo de las ciencias sociales en Europa, y su programa de intervención se centraba en el desarrollo económico y social de América Latina y Asia, y aconsejó que se dirigieran a la Fundación Ford. Con esta fundación se había puesto ya en contacto el director general de la Enseñanza Superior, Gaston Berger, para discutir con los responsables de las universidades americanas un proyecto de 
reorganización de la enseñanza superior francesa. Berger trató de ganarse la voluntad de la Fundación para un conjunto de proyectos sobre ciencias humanas, entre los que se encontraba un centro universitario internacional, pero los responsables de la Fundación le hicieron saber que se interesaban más por proyectos limitados en el tiempo o por bibliotecas como la de ciencias sociales, sobre todo en un país donde las condiciones de trabajo documental que se ofrecían a los investigadores eran muy deficientes.

Estos proyectos oficiales coincidieron con los del grupo de Braudel, que pedía la creación de un organismo que favoreciese la comunicación entre investigadores de diferentes centros y racionalizase los servicios técnicos y de documentación. Esta idea gustó más a la Fundación Ford, que recalcó su interés por influir en el carácter de la Maison, precisando que la orientación que querían dar a su ayuda era "el progreso rápido de la industria francesa de la posguerra y acelerar el proceso de modernización que requerían economistas, sociólogos y otros especialistas en ciencias sociales» ${ }^{16}$.

El acuerdo que llevó a constituir la Maison en 1957 obtuvo un millón de dólares de la Ford y dos millones del gobierno francés.

En el Reino Unido la Fundación Rockefeller tenía ya una larga tradición de ayuda económica, no sólo a las grandes universidades como la London School of Economics (LSE), sino también a colleges y bibliotecas como las de Oxford y Cambridge. En el caso de la sociología, cuyo primer núcleo de posguerra se formó en la LSE, los viejos amigos de la fundación dieron fondos para la coordinación de la ciencia social y para investigar sobre temas de población y movilidad social, cantidades que se unieron a las aportadas por otras fundaciones inglesas como la Nuffield y a donaciones privadas ${ }^{17}$. La colaboración de la Rockefeller tuvo muchas ramificaciones en otras universidades inglesas.

Esta fundación volcó también sus esfuerzos en Alemania, donde contribuyó decisivamente a la financiación de investigaciones aplicadas a la industria, a la creación de la Universidad Libre de Berlín y a la vuelta del Instituto de Frankfurt.

En este último caso el Instituto había cortado sus lazos con la Universidad de Columbia en 1946 y el primer viaje de su director M. Horkheimer a Europa se produjo en 1948, cuando fue becado por la Rockefeller para ser profesor invitado en la Universidad de Frankfurt. Allí inició sus primeros contactos para organizar la vuelta del Instituto que se produjo en 1950, cuando el comisario americano McCloy puso a su disposición 200.000 marcos para actividades académicas y 235.000 para su reconstrucción. Esta gran ayuda nacía de la convicción que había entre los responsables americanos de la política en Alemania de que la sociología, particularmente cuando estaba representada por ciudadanos americanos con su énfasis sobre la investigación empírica, era un factor de promoción y desarrollo de la democracia. De hecho, Horkheimer,

16. Ibídem, p. 142-157.

17. DAHRENDORF, R. (1995). A history of the London School of Economics and political science 1895-1995. Oxford: Oxford University Press. 
para asegurar las finanzas, enfatizó la participación del Instituto en la reconstrucción de la posguerra, y en un memorándum que entregó a los patrocinadores ofrecía no sólo un curso progresivo de estudios en sociología que «conectaba la tradición alemana de la filosofía social y las humanidades con los métodos avanzados de investigación empírica de la sociología americana moderna, sino también un centro que ofrecía asesoramiento académico sobre los problemas urgentes del Estado» ${ }^{18}$. Esta actitud provocó la reacción de L. Löwenthal, su colaborador y amigo, quien le recriminó por escrito, pero Adorno respondió que él era partidario de la independencia individual y de que la ciencia se liberase de sus anteojeras.

La Fundación también financió otros proyectos y departamentos de sociología, como el Área de Investigación Sociológica de la Universidad de Münster, dedicada prioritariamente a la sociología industrial, una de cuyas grandes investigaciones sociológicas, dirigida por H. Popitz en 1953-54, fue la influencia de los aspectos sociales y tecnológicos sobre el trabajo social en la industria del hierro y el acero, a la que siguió una segunda parte que trataba sobre la imagen que tenían los trabajadores de su propio trabajo, del progreso tecnológico, de su participación en el proceso productivo y de la sociedad en general.

Fue una época en la que la ciencia en Alemania tuvo muchas ayudas del gobierno americano y de fundaciones privadas, de tal manera que entre 1948 y 1954 alrededor de once mil alemanes habían visitado Estados Unidos, sobre todo empresarios, académicos, profesionales del derecho, de los medios de comunicación y estudiantes.

Uno de los centros de nueva construcción fue la Universidad Libre de Berlín, que se inauguró en 1948 y se configuró como un modelo de referencia para el resto de los centros académicos del país. Su estructura democrática, la participación de los estudiantes en la vida y la administración de la universidad y el espíritu comunitario que existió desde su nacimiento fueron características que la distinguían del resto de las universidades alemanas, organizadas todavía con ideales autoritarios y jerárquicos típicos de los años de la preguerra.

Los americanos tuvieron una relación especial y un contacto muy estrecho con la Universidad Libre y aunque dos terceras partes de su financiación venían del gobierno de Bonn y de la propia ciudad de Berlín, el gobierno americano y la Fundación Ford concedieron muchas ayudas para subsidiar la construcción de clínicas médicas, bibliotecas y un instituto de estudios americanos. Para los americanos esta universidad era producto y símbolo de la Guerra Fría. Sus batallas anticomunistas y su compromiso con los valores democráticos eran un antídoto contra el totalitarismo de la Alemania oriental ${ }^{19}$.

La única sociología europea que permaneció al margen de todas estas ayudas fue la italiana que, no obstante, encontró su primer cobijo en la Fundación 
Olivetti. En la formación del primer núcleo de la sociología italiana tuvo un papel fundamental el movimiento social e intelectual creado alrededor de las industrias Olivetti de Turín. Adriano Olivetti era un intelectual turinés, medio judío medio protestante, vinculado a los circulos de la Resistencia que combinaba un interés personal en la comunidad con una apertura intelectual y práctica a los modelos extranjeros de modernización industrial. Su movimiento fue una continuación de las ideas del Partido de Acción, un partido intelectual de izquierdas que no sobrevivió a las elecciones democráticas de 1948. Este movimiento proponía la unión entre ciudadanos, profesionales y políticos para la creación de una base democrática articulada e integrada por células de grupos pequeños y concienciados en la defensa de la libertad, la democracia y la justicia en armonía de intereses. Su revista Comunità era la única plataforma cultural de posguerra donde podían debatirse las nuevas ideas para el cambio social y la modernización. El centro de estudios Olivetti hizo planes reguladores para la ciudad donde se ubicaban sus empresas y llamó la atención de algunos intelectuales que querían estudiar la realidad social con una perspectiva práctica que fuese más allá de las generalizaciones marxistas sobre la clase obrera, ofreciendo trabajo a los primeros sociólogos de posguerra como F. Ferrarotti, A. Pizzorno o L. Gallino, que no tenían otras estructuras de apoyo, ni estatales ni privadas ${ }^{20}$.

\section{Funcionamiento y orientación científica}

Ya hemos visto que, según Turner, la decisión de financiar proyectos de investigación en estas instituciones era muy personal y que se utilizaban pocos mecanismos de control, ni siquiera la revisión formal de las propuestas por parte de otros colegas, con lo cual se desarrolló un vínculo personal entre los burócratas de estos centros y el mundo académico, lo que permitía que la financiación de los proyectos se aprobase sin demasiadas dificultades. Esto influyó considerablemente en la clasificación y el prestigio de las universidades americanas y, sobre todo, en la preeminencia de las grandes universidades privadas sobre las públicas, que no tenían tantos recursos ni podían competir con las primeras.

Además, los directores de las fundaciones en los años cincuenta constituían una comunidad profesional, con su propia red ocupacional y sus niveles jerárquicos. Era común entre ellos trasladarse de una institución a otra, y los representantes de las fundaciones tenían reuniones y encuentros sobre temas de interés común, incluso algunas veces hacían tratos acerca de la división del trabajo en sus organizaciones y en las áreas de especialización donde sus éxitos debían ser reconocidos. De esta manera, las redes universitarias y de financiación se solapaban con los intereses académicos, lo cual era importante para los

20. PINTO, D. (1997). «Sociology as a cultural phenomenon in France and Italy». Ph. D. Harvard University. 
directivos de las fundaciones que se identificaban más con estos académicos que con los intereses de los fundadores. Aún así, en general había un buen nivel de consenso y legitimación mutua, de tal manera que un estudio de Berelson realizado en 1956 indicó que la mayor parte de las ayudas concedidas entre 1930 y 1956 se había distribuido entre los más cualificados.

Sin embargo, no todos estaban de acuerdo con este tipo de mecanismos para adjudicar los proyectos y de hecho algunos se quejaban de que las ayudas de las fundaciones recayeran casi siempre sobre un pequeño grupo de académicos. Otro problema añadido era el aumento de la gestión, de tal manera que la Ford, que había tomado el protagonismo después de la guerra, se quejó de que los profesores que han de presentar un proyecto quedan sumidos dentro de la burocracia y de las luchas entre académicos y burócratas abandonando la enseñanza e investigación para dedicar su tiempo a pedir ayudas económicas. Quizá habría que volver al sistema de que fuese la fundación la que eligiese un tema importante y lo encargase a un director o a un equipo de investigadores, como había ocurrido en el caso de G. Myrdal —El dilema americano - o más tarde en el de D. Riesman con La muchedumbre solitaria (1950), en los que la fundación Carnegie había utilizado esta fórmula con gran éxito ${ }^{21}$.

Ahora bien, el tipo de investigación que se impuso en la mayor parte de estos estudios como orientación metodológica fue el survey, un trabajo psicosocial exclusivamente cuantitativo y muy rutinizado, impulsado por el éxito del Bureau of Applied Social Research que dirigió durante muchos años P. Lazarsfeld. Este tipo de investigación tuvo su máximo predicamento y expansión durante la guerra, aprovechando el interés del gobierno sobre la moral del ejército y de la población. En esos años se crearon tres centros de investigación oficiales dependientes del ejército: el Research Branch of the Division of Moral of the U.S. Army, bajo la dirección de S. Stouffer; el Survey Division of the Office of War Information, dirigido por D. Wilson, y por último el Program Survey of the U.S. Department of Agriculture, dirigido por R. Likert. Algunos de estos sociólogos o miembros de sus equipos fundaron oficinas $\mathrm{u}$ organizaciones de survey después de la guerra en otros centros y universidades americanas: R. Likert en Michigan, S. Stouffer en Harvard, etc. ${ }^{22}$.

Las fundaciones desempeñaron también un papel fundamental en el desarrollo de la investigación social cuantitativa y en la difusión de sus métodos y técnicas científicas. La producción de artículos empíricos creció en Estados Unidos desde el 38\% en 1920 al 67\% en 1930 y más del 80\% después de la guerra. A la teoría apenas se le dejó espacio, aunque la Ford llevaba a cabo un programa de investigación teórica en 1955 bajo la dirección de Talcott Parsons, y la Carnegie financió su Teoría General de la Acción. Pero las fundaciones fueron las primeras en suscitar artículos empíricos en los años veinte y treinta sin dar apenas subvenciones a los trabajos teóricos ${ }^{23}$.

21. Turner, S.P.; TURner, J.H., op. cit., p. 90-100.

22. Ibídem, p. 100-104.

23. Platt, J., op. cit., p. 168-169. 
Hemos visto, pues, que estas instituciones privadas impulsaron programas de reforma, se dedicaron a la investigación social aplicada y financiaron proyectos de acuerdo con sus directrices. La Rockefeller tuvo el protagonismo más importante y fue la que dedicó más recursos, seguida de la Carnegie Corporation, cuyos recursos se aplicaron en buena medida a iniciar programas de estudio en las universidades más importantes, siendo uno de los más significativos el Russian Research Center, que recibió el 23\% de los fondos de la fundación en este campo. De este importante centro fue director C. Kluckhohn y en él trabajó T. Parsons. Pero también dedicó cantidades sustanciales para la promoción del intercambio internacional ${ }^{24}$, y en el caso de la sociología financió algunos proyectos muy importantes como American Soldier, dirigido por Stouffer, a quien apoyó después para fundar el Laboratory of Social Relations de Harvard.

Sin embargo, después de la Guerra tanto la Fundación Rockefeller como la Carnegie perdieron protagonismo en favor de la Ford, que se había constituido en 1936 en Detroit, donde permanecería hasta 1950. A partir de esta fecha aumentó considerablemente los recursos dedicados a las ayudas, tanto dentro como fuera de Estados Unidos, de tal manera que hacia los años sesenta superaban los doscientos millones de dólares al año y a finales de 1975 habían sumado un total de cinco billones, con pagos hechos a más de siete mil instituciones y organizaciones en los Estados Unidos y en ochenta y tres países extranjeros $^{25}$.

La tarea a la que Ford dio un gran impulso en los años cincuenta y que mayor impacto causó en la ciencia social, sobre todo en la ciencia política, fue el desarrollo de un programa nacional de apoyo al conocimiento y puesta en práctica del estudio del comportamiento en el ámbito político (behaviorismo), que alcanzó su cenit cuando R. Dahl publicó en 1961 su artículo «The Behavioral approach to political science: Epitaph for a movement to a succesful protest» en la American Political Science Review, cuyo método se extendió rápidamente como una forma nueva de hacer ciencia y de leer la realidad sociopolítica distinta a la historia, la economía e incluso la politología tradicional. El programa comportamentista incluía la psicología, la antropología, la sociología y materias muy relacionadas con tales disciplinas como la biología o la psiquiatría, que trataban de entender el voto a través de encuestas de opinión y cuestionarios.

El proyecto se puso en marcha cuando un comité comenzó a discutir sobre los problemas del bienestar social en Estados Unidos y la apatía política de los ciudadanos. Estos hechos, junto al temor de un retorno a la economía deprimida de los años treinta y al convencimiento de que el sistema educativo no

24. GeIger, R.L. (1988). «American Foundations and academic social science 1945-60». Minerva, 26, p. 315-341.

25. SEYBOLd, P. (1987). "The Ford Foundation and the transformation of political science». En SChWARTZ, M. (ed.). The structure of power in America. Nueva York: Holmes and Meier, p. 185-197. 
estaba asentado en un país que había estado sometido a cambios muy rápidos en poco tiempo, eran motivo de preocupación. La Ford creó entonces un área para investigar el comportamiento humano de forma científica. La creación de esta área (The Center for Advanced Studies in the Behavioral Sciences) fue una idea propuesta por Lazarsfeld y Merton dada su experiencia en el Bureau for Applied Social Research, pero tambien evidenciaba la creencia en que las ciencias del comportamiento podían contribuir de manera decisiva a la solución de los problemas sociales básicos en América, ya que los politólogos tradicionales no podían afrontar esta tarea, porque no tenían una orientación empírica. Así pues, entre 1951 y 1957, la Behavioral Science Division recibió más de veintitrés millones de dólares, que contribuyeron no sólo a la investigación, sino también a la formación de especialistas extranjeros para que estudiasen en la práctica los mecanismos del comportamiento humano.

Para los defensores de esta metodología sólo la adquisición de informaciones, datos, números y estadísticas cuidadosamente controlados podían permitir que la ciencia política se caracterizase como policy science, es decir, como una forma de conocimiento capaz de influir, de manera directa e incontrovertible, en la actividad de los políticos y en las decisiones del gobierno y de la administración, tanto a nivel local como internacional.

Al proponer al individuo como el centro de la experiencia política y consiguientemente de la investigación politológica, el comportamentismo no solamente deja de usar conceptos y categorías de origen ochocentesco, sino que introduce en el análisis político un vocabulario nuevo cuyos términos más frecuentes son: condiciones y condicionamientos; percepciones y aptitudes; identidad y comunicación; motivaciones y persuasión; adaptación e integración; ventajas y privaciones; participación y apatía.

D. Easton es uno de los estudiosos que mejor han sistematizado las bases de esta corriente comportamentista en su trabajo «A framework for political analysis» (1965), donde describe los presupuestos comunes a todos sus representantes, que resume en ocho proposiciones de carácter epistemológico: 1) regularidad; 2) control; 3) técnicas; 4) cuantificación; 5) valores; 6) sistematización; 7) ciencia pura, y 8) integración.

G. Sola ${ }^{26}$ ha resumido estas proposiciones comentando que la primera se refiere a la aceptación del principio de regularidad, según el cual en el comportamiento político se pueden individuar uniformidades que se expresan bajo forma de generalizaciones empíricas o como teorías dotadas de valor explicativo y predictivo. El segundo elemento subraya la centralidad de la observación. La validez de generalizaciones y teorías nace no tanto de especulaciones o deducciones abstractas cuanto de una observación constante de la realidad sometida a control y, por tanto, verificada en referencia a comportamientos y fenómenos empíricamente comprobados. El tercer elemento reclama la necesidad de 
las técnicas de verificación. La validación o el desmentido de las teorías se apoya sobre instrumentos rigurosos específicamente predispuestos para observar, registrar y medir el comportamiento. El cuarto elemento se refiere a la cuantificación, es decir, a la utilización de técnicas adecuadas en la medida y presentación de los datos del observador. El quinto punto, referido a los valores, sostiene que los valores morales y las explicaciones empíricas son distintos y deben dar lugar a proposiciones también distintas. La sistematización exige la necesidad de una relación estrecha entre teoría e investigación como partes conectadas en un conjunto cognoscitivo coherente y ordenado. El séptimo punto sostiene que la ciencia pura ha de preceder a la aplicada, y, por último, el octavo subraya que la ciencia política debe asumir los conocimientos de las otras ciencias del comportamiento si no quiere debilitar la validez de sus hallazgos.

Se trata, para muchos, de un enfoque mecanicista que, además de asumir como unidad de análisis al individuo particular y privilegiar los métodos cuantitativos y de control experimental, enfatiza el condicionamiento de la acción hasta eliminar cualquier margen de libertad o elección personal. Los portavoces de este nuevo paradigma que en los años cincuenta hablan de la necesidad y la urgencia de introducir el método científico en la investigación política son G. Almond, R. Dahl, H. Simon, S.H. Barnes y M. Janowitz, entre otros. Con ello se separan del modo tradicional de hacer ciencia política, que parte de una teoría general del Estado y del poder, a menudo normativa, y reconducen el poder al hecho de participar en la toma de decisiones, exaltando la dimensión "voluntarista» del proceso político, desconociendo o ignorando también la eficacia condicionante de las estructuras, que es un elemento clave en los análisis de inspiración marxista.

La Fundación Ford animó también a crear programas diseñados para desarrollar la teoría política comportamentista en otras instituciones. En las universidades de Columbia (Lipset, Hyman), Chicago y otras se desarrollaron grupos y seminarios para trabajar en la investigación del comportamiento político y social. La Ford dio 36.000 dólares al National Opinion Research Center y la Rusell Sage Foundation recibió 750.000 para desarrollar un programa para la utilización práctica de las ciencias del comportamiento. El último esfuerzo se dirigió al SSRC y a sus comités de estudio sobre el comportamiento político y las políticas comparadas que apoyaron investigaciones en el ámbito internacional, nacional y local. Libros, artículos y revistas comenzaron a difundir la nueva ciencia política comportamentista que empezaba a dominar la literatura en este campo. Para algunos estudiosos esto fue una prueba evidente de la influencia que ejercieron las fundaciones sobre la producción de ideas y su cambio en la sociedad. La ciencia política había cambiado para acudir en apoyo de la elite económica.

\section{Las fundaciones en el ámbito de la política cultural americana}

El papel de las fundaciones en el ámbito de la política cultural y científica americana ha tenido diversas lecturas, y su relación con la sociología ha sido inter- 
pretada de diversas maneras. Del lado americano J. Platt ${ }^{27}$ ha resumido algunas versiones, pero ha olvidado contribuciones europeas importantes, como las de P. Bourdieu o M. Pollak. Antes de pasar a los aspectos más críticos, diremos que algunos autores, I.L. Horowitz, S.Z. Klausner, R. Pells, sitúan su actuación política dentro de lo que fue el desarrollo de la sociedad y la cultura americanas en la primera parte del siglo XX y sobre todo de la repercusión que tuvieron en ella y en el papel del Estado las dos guerras mundiales. Para Horowitz, las fundaciones tuvieron un papel de apoyo al Estado y a sus políticas públicas, tanto dentro como fuera de América, que a su vez sirvió para integrar y legitimar a las ciencias sociales y a sus científicos en el marco de los postulados liberales. Comparando la relación de la sociología con la sociedad en un estado totalitario, en un estado del bienestar o en un sistema liberal, considera que en Europa el Estado del bienestar ha sido un motor para el crecimiento de la ciencia social y a su vez las ciencias sociales han ido conformando las tendencias socialistas. En países como Inglaterra, Francia o Alemania antes de introducir una ley determinada era muy probable que se llevase a cabo un sondeo (survey) sobre la opinión pública en ese ámbito. Después de la Segunda Guerra Mundial esta política se ha extendido de manera general, y la tendencia reformista dedicada a la eliminación de la pobreza, la integración de las minorías étnicas o el equilibrio del desarrollo urbano llevó también a las fundaciones filantrópicas más importantes a ocuparse de estos problemas ${ }^{28}$.

Quizá por eso la Administración pública americana y los diferentes gobiernos se habían mostrado siempre reluctantes a tener un departamento propio de ciencias sociales dentro de su política científica. Ahora bien, el Congreso, viendo los resultados que había dado el conocimiento técnico en la guerra y el papel que algunos trabajos de ciencias sociales (American Soldier...) habían tenido en ella, estaba convencido de que la ciencia se había mostrado crucial para la victoria y que desarrollaría un papel muy importante en el poder mundial del futuro. Por eso se cuestionaron el papel que debería tener la ciencia social en la recientemente creada National Science Foundation.

¿Cuál es el uso que se ha de dar a las ciencias sociales en el interés nacional?, ¿cuáles han sido sus logros hasta el momento presente?, ¿cuál es su lugar en la sociedad industrial moderna y sus perspectivas de futuro? Preguntas que podían resituar el papel del Estado y el de las fundaciones respecto a las ciencias sociales. Así fue como, segun S.Z. Klausner ${ }^{29}$, en el ámbito del Social Science Research Council, el gobierno federal formó un comité que tomó parte en la planificación nacional de las ciencias después de la guerra y nombró, en 1945, a Talcott Parsons para que redactase un documento sobre el papel que deberían tener en el futuro las ciencias sociales, a fin de promover el apoyo gubernamental.

27. Platt, J., op. cit., p. 142-199.

28. HorowITZ, I.L., op. cit., p. 38-68.

29. Klausner, S. Z. (1986). "The bid to nationalize american social science». En KLAUSNER; LIDZ, op. cit., p. 3-41. 
Parsons publicó una primera versión en 1946 y entregó su manuscrito, «Ciencia Social: un recurso nacional básico», en 1948, pero los miembros del comité entendieron que el informe no respondía a lo que ellos habían pedido al autor. Más que un escrito para favorecer el apoyo gubernamental a las ciencias sociales, se trataba de un texto de teoría social, su relación con la investigación social empírica y la contribución de la ciencia social a la formación de la política nacional doméstica y extranjera.

En esta tarea de oficializar el estatuto de la sociología, Parsons se enfrentó, por una parte, a quienes en el SSRC creían que la ciencia social debía ser apoyada por universidades competentes e instituciones privadas de investigación que siempre respetarían más la libertad de juicio de los estudiosos, puesto que la ayuda del gobierno podía convertir a los investigadores en sus portavoces, y, por otra, a quienes pensaban que las ciencias sociales formaban parte de la cultura general pero no eran verdaderas ciencias, sin olvidar tampoco el obstáculo de aquellos senadores de la América profunda que por ciencias «sociales» entendían ciencias «socialistas». En esta batalla trató de persuadir a los científicos experimentales de la necesidad de incluir las ciencias sociales en la National Science Foundation, con el fin de promover la utilidad de las ciencias humanas para la seguridad de la defensa nacional y el avance de la salud y el bienestar; Parsons subrayó que «el potencial de la ciencia social está en su utilidad», y anticipó una «tecnología social» comparable en finalidad y productividad a la ciencia experimental aplicada. Sus argumentos enfatizaban la unidad del método en las ciencias y su oposición a que la ciencia social se utilizase como ideología para el cambio social o al servicio de la crítica moral a la tecnología. El diagnóstico sobre la eficacia de las organizaciones, los análisis de la opinión y las actitudes, la recogida de datos a gran escala y otros aspectos sociales del estudio de la población requieren fondos federales. Contrario a la Escuela de Chicago, defendía que la sociología debía utilizar conceptos abstractos y adherirse a normas de neutralidad ética. En esto, Parsons, que ya había publicado La estructura de la acción social (1937), era coherente con su pretensión de formular un programa teórico de categorías sociales que estableciese una relación con la realidad concreta.

Pero en todo ese tiempo el Congreso no aprobó nunca una ley que apoyase a las ciencias sociales. Sólo a partir de 1960 el Office of Social Science adquirió el rango de área independiente dentro del NSF de la mano del sociólogo H. Alpert. Según J. Riley ${ }^{30}$, que fue colaborador suyo cuando la Fundación Ford entró en el proyecto, Parsons estaba convencido de que la ciencia social era una parte esencial de la sociedad y tendría un interés creciente, pero sus ideas fracasaron por tres razones: por la debilidad del estatuto político de las ciencias sociales, por el escepticismo que muchos profesaban acerca de su naturaleza científica y por el criterio de algunos de sus valedores de que las ciencias sociales eran menos importantes que otras para los temas que estaban en

30. RiLeY, J.W. (1986). «The status of the social sciences, 1950: A tale of the two reports». En KLAUSNER; LiDZ, op. cit., p. 42-113. 
juego, cuyas disciplinas habían producido el radar, la penicilina, la bomba atómica y otras contribuciones importantes del éxito militar más reciente.

Este retraso de la Administración pública para apoyar las ciencias sociales en general y la sociología en particular permitió que las fundaciones protagonizasen la investigación en este campo hasta la década de los sesenta.

Por otra parte, R. Pells $s^{31}$, que ha escrito más directamente sobre la relación Europa-USA en esa época, considera que la política de las fundaciones se debe entender dentro del marco general de lo que fue la política cultural americana después de la Segunda Guerra Mundial en su lucha ideológica contra el comunismo y su necesidad de penetrar en las principales instituciones científicas europeas. Según este autor, su actuación fue una alianza entre la cultura americana y la política exterior de Washington, que empezó con la guerra y se expandió después durante la Guerra Fría.

En este periodo la Fundación Rockefeller fue la primera en financiar la emigración intelectual a Estados Unidos, con el 50\% de las ayudas, aunque su asistencia se dirigió solamente a los estudiosos senior más estabilizados quienes no deberían competir por la promoción académica con los jóvenes americanos que estuviesen empezando sus carreras. Concedió una ayuda de 540.000 dólares a la New School for Social Research, que fue el centro que acogió la mayor concentración de emigrantes intelectuales extranjeros.

El Plan Marshall, que duró de 1948 a 1952 y cuya finalidad declarada era la recuperación económica europea, tenía también un componente cultural que consistió en vehicular los valores de la libertad de empresa, sindical y comercial, así como los principios de la productividad, la competencia, la eficacia y el bienestar a través de películas y documentales, programas de radio, pósters y todo tipo de expresión gráfica. Había que difundir un estilo de vida americano en todas sus facetas. Se lanzó un vasto programa de ayudas a la investigación, tanto privada como pública. Las becas Fulbright estuvieron vinculadas desde el principio al Departamento de Estado y de ellas se beneficiaron, Inglaterra, Francia y Alemania.

Recordemos, finalmente, que la Guerra Fría se caracterizó por la política del presidente $\mathrm{H}$. Truman, que la describió como una «lucha, sobre todo, por la mente de los hombres» y más ideológica que bélica, y en ese proyecto trataron de conquistar la voluntad de algunos de los intelectuales europeos más prominentes, como ocurrió en los casos de Aron o Rusell.

\section{Los fondos estatales sustituyen a las fundaciones}

Convendría recordar en este contexto que las primeras ayudas a la investigación sociológica que concedió el gobierno americano fueron dirigidas a la agricultura a comienzos del siglo. Eran trabajos dedicados a proyectos de cooperación dentro del Ministerio de Agricultura a través del Bureau of the Census 
y la Division of Farm Population and Rural Welfare, que aumentaron de volumen durante el periodo del New Deal. Se trataba de recoger datos sobre la población y las opiniones de los agricultores. Fue en este periodo cuando se contrató a R. Likert, que perfeccionó la metodología y las técnicas aplicadas tanto cuantitativa como cualitativamente. Por eso en algunos países, no sólo en Estados Unidos, la tradición de la sociología agraria ha constituido una de las bases de la sociología posterior, sobre todo en su proyección más aplicada y sociográfica ${ }^{32}$. Recordemos que R. Dahl antes de entrar en el mundo académico (1946) había colaborado con el Ministerio de Agricultura.

Ya hemos dicho antes que se le dio un nuevo impulso a la sociología con el New Deal y todos los planes de reforma, incluyendo los proyectos de la Administración para el mundo del trabajo (Work Projets AdministrationWPA). Por primera vez se posibilitó que muchos sociólogos recogiesen datos a gran escala y se empleasen muestras y métodos estadísticos aplicados a problemas reales y concretos de la sociedad ${ }^{33}$.

El Social Science Research Council (SSRC) tuvo como primera finalidad la transformación de las ciencias sociales, pero en los años treinta su objetivo se desvió hacia la reforma de la administración gubernamental. La Depresión inclinó la balanza de su apoyo hacia los temas políticos relevantes, con lo cual la sociología perdió peso y el dinero que se le dedicaba se orientó en esos años a trabajos posdoctorales a los que concursaron H. Blumer, S. Stouffer, L. Wirth, etc.

También hemos visto que la guerra dio pie al nacimiento de diversas unidades de investigación social que tuvieron gran repercusión en el desarrollo posterior de la disciplina. Pero, aún así, el Gobierno Federal nunca dio un apoyo considerable a la sociología a pesar del esfuerzo que hizo Parsons con su informe para incluirla en una de las áreas de la National Science Foundation. Ninguno de los ejercicios posteriores a 1950 la incluyó en su presupuesto.

Sólo a finales de la década de los cincuenta ${ }^{34}$, cuando la superioridad americana se puso en cuestión por la maquinaria soviética, los fondos federales experimentaron un gran aumento, dirigidos, sobre todo, al sistema educativo y a las ciencias básicas, pero beneficiándose también las ciencias sociales. Respecto a estas últimas, el presupuesto del Gobierno Federal entre 1956 y 1980 aumentó desde 30 millones de dólares a 424 millones, y las fundaciones privadas dieron un gran salto entre 1946 y 1958 en su dotación a las universidades tanto públicas como privadas ${ }^{35}$ (tabla 1 ).

De la suma total (85.767), la Carnegie había contribuido con 14.799 millones de dólares; la Rockefeller, con 9.797, y la Ford, con 61.171. Además, estas tres fundaciones habían dado al Social Science Research Council 12.049 millones.

32. Platt, J. (1986), "Qualitative research for the state». Quaterly Journal of Social Affairs, 2, p. $87-108$.

33. CONVERSE, J.M. (1987), Survey research in the US: Roots and emergence 1890-1960. Berkeley: University of California Press.

34. Turner, S.P. ; TURner, J.H., op. cit., p. 133-147.

35. GEIGER, R.L., op. cit., p. 333-335. 
Tabla 1. Ayuda de las fundaciones Carnegie, Rockefeller y Ford a las ciencias sociales en diecisiete universidades, 1946-1958 (en miles de dólares).

\begin{tabular}{lr}
\hline Universidades & Ayuda \\
\hline California & 3.941 \\
Illinois & 1.079 \\
Michigan & 4.380 \\
Minesota & 1.892 \\
N. Caroline & 1.706 \\
Wisconsin & 987 \\
Chicago & 11.714 \\
Columbia & 12.226 \\
Cornell & 4.005 \\
Harvard & 17.530 \\
John Hospkins & 1.626 \\
MIT & 5.322 \\
Northwestern & 1.705 \\
Pennsylvania & 2.966 \\
Princeton & 3.705 \\
Stanford & 4.677 \\
Yale & 6.206 \\
\hline
\end{tabular}

La sociología y los sociólogos se popularizaron porque tuvieron además una presencia física en los medios de comunicación de masas, tanto en la prensa como en la televisión. T. Parsons, C.W. Mills y otros conocidos representantes de la disciplina trataron de hacer llegar al gran público una lectura de la sociedad que ponía de manifiesto sus deficiencias y disfunciones. Los problemas sobre la educación, la familia, la pobreza o el crimen eran patentes y la conclusión que se sacaba en muchos casos era que el Estado debía intervenir. Buena parte de la opinión pública vinculó la sociología con la denuncia de la sociedad y su reforma, una lógica que fue utilizada para sacar dinero del gobierno y de las fundaciones privadas con el fin de estudiar y conocer mejor los problemas sociales, uno de cuyos efectos fue el crecimiento de la estructura profesional, la valoración de la carrera académica y, en general, la consagración de la sociología como disciplina científica con el mismo estatus que el resto de las disciplinas sociales.

\section{La crítica a las fundaciones}

Con el transcurso de los años el papel de las fundaciones ha sido sometido a crítica desde ángulos muy diversos y también desde diversas geografías. El panorama crítico ha sido más rico y complejo en Estados Unidos, mientras que en 
Europa el debate se polarizó en Francia e Italia, sobre todo por la fuerte presencia de los partidos comunistas, ya que Inglaterra y Alemania se vieron ajenas a esta polémica.

En Estados Unidos las críticas moderadas vinieron de A. Gouldner ${ }^{36}$, D. Fisher ${ }^{37}$ y I.L. Horowitz ${ }^{38}$, mientras que las más duras llegaron del maccartysmo por la derecha y de quienes vieron mezclados con las fundaciones intereses mucho más espúreos, como S. Diamond o F.S. Sanders por la izquierda.

A. Gouldner argumentó que tanto las fundaciones como el Estado, que representaban los intereses de las elites económicas y políticas, ejercieron un fuerte control sobre la ciencia social, de tal manera que esto condicionó tanto los métodos y las técnicas como los resultados de la investigación. El aumento del instrumentalismo priorizó los métodos positivistas en detrimento de la teoría, puesto que se fijó casi exclusivamente en aquellos problemas y en aquellas variables que le interesaban a la Administración pública. Para Fisher, además, el matrimonio entre científicos y Administración fue lo que fortaleció y legitimó la profesión que se puso al servicio de los intereses del capital, esgrimiendo una neutralidad metodológica y valorativa que les situaba más allá de todo interés particular o de grupo. Las fundaciones se habían mostrado colaboradoras muy activas de la ideología dominante y del poder establecido. Para Horowitz, sin embargo, la cuestión está en que en los sistemas de bienestar la política y las ciencias sociales interactúan sin ningún sentido de la tensión o contradicción entre las proposiciones científicas de unos y las orientaciones terapéuticas de los otros. La integración, en este caso, es tan completa que se produce una pérdida de identidad tanto en el polo científico como en el político. Todas las funciones de la ciencia social están encaminadas a la orientación de los problemas sociales, cuyo resultado es el declinar del interés en análisis más amplios de las fuerzas y los sistemas sociales, es decir que se produce una reducción de la autonomía de la ciencia social. Teóricamente, las fundaciones sin ánimo de lucro deberían animar la investigación pura, pero tendieron a interesarse por los problemas prácticos para justificar el mérito de sus donaciones ante los hombres de negocios y las instancias gubernamentales.

El hecho de que el presidente de los Estados Unidos pueda participar indirectamente en el proceso de selección de los directivos de las fundaciones más importantes indica la íntima conexión que existe entre los administradores públicos y privados de la riqueza. Todo esto contribuye a que quienes más utilizan la investigación son el gobierno federal, las industrias básicas y la publicidad, y los que menos, los pobres y las minorías, por eso la ciencia social es un reflejo suave de la riqueza. Por tanto, los científicos sociales, en la medida en que se comprometen con las instituciones que hacen política (fundaciones,

36. Gouldner, A.W. (1979). La crisis de la sociología occidental. Buenos Aires: Amorrortu.

37. FISHER, D., op. cit., p. 206-233.

38. HorOWITZ, I.L., op. cit. 
etc.), se comprometen con una ideología elitista, porque aceptan que quienes cambian las cosas son los de arriba. Por eso las necesidades de la política es difícil que cuadren con las aporías y necesidades de la ciencia social.

Desde otra perspectiva, Bulmer, que ha estudiado de cerca la vida y el funcionamiento de la fundación Rockefeller, argumenta que el apoyo considerable que dio a la sociología permitió a los sociólogos escoger los temas según sus propias prioridades intelectuales, y además posibilitó un trabajo cuantitativo sin el cual los profesionales no hubiesen podido obtener recursos para recoger datos a gran escala y compilar estadísticas. J. Platt piensa, a su vez, que el capitalismo y sus instituciones podían haber utilizado sus fondos de muchas otras maneras para potenciar su imagen y buscar su legitimación sin necesidad de crear bibliotecas públicas (Carnegie), erradicar enfermedades (Rockefeller) o apoyar y hacer campañas por los derechos civiles (Ford).

Más a la derecha, las fundaciones fueron criticadas por los aspavientos del maccartysmo, que se dejaron sentir sobre todo en la Rockefeller y su política europea. Las fundaciones eran consideradas por la extrema derecha como instituciones potencialmente subversivas, que promueven valores antiamericanos como el internacionalismo y el colectivismo, y apoyan a elementos comunistas. En su ceguera atacaron incluso a Stouffer por proporcionar datos que se utilizaron, según ellos, para desmovilizar al ejército después de la Segunda Guerra Mundial, lo que delibitó a la defensa americana ${ }^{39}$. Lo que ahora nos parece histriónico en los años cuarenta y cincuenta obligó a los responsables de estas instituciones a hacer restricciones. Una campaña de prensa las acusó de utilizar fondos exentos de impuestos para la financiación de propaganda comunista. La fundación Rockefeller, que no había dado ninguna consigna interna para discriminar políticamente a sus candidatos, fue acusada de becar científicos y artistas comunistas, lo cual paralizó una ayuda ya concedida de 350.000 dólares para el desarrollo de los laboratorios franceses del CNRS y suspendió sus relaciones con este organismo mientras no fuesen sustituidos algunos de sus representantes comunistas. Con este motivo, la fundación abrió un debate sobre este tema, y llegó a la conclusión de que su responsabilidad moral era encontrar una solución intermedia entre el maccartysmo y el sostenimiento de Stalin, puesto que ellos no eran un brazo del $\mathrm{FBI}^{40}$.

En este contexto la creación de la VI Sección de Ciencias Sociales en Francia encontró algunos obstáculos, aunque Braudel supo resolver la situación evitando la discriminación política e instaurando la pluralidad ideológica, para demostrar el carácter universitario y no político del proyecto, tanto frente a ellas como frente a los comunistas franceses que les tachaban de "vendidos a los americanos». Una acusación que también los comunistas italianos lanzaron al proyecto Olivetti, al que tildaban de estilo americano, que utilizaba métodos paternalistas para dividir a una clase obrera subdesarrollada. 
Desde el ángulo opuesto, investigaciones recientes han puesto de relieve el entramado sociopolítico que se formó en momentos difíciles, tanto en las fundaciones como en las universidades, y han propiciado una crítica más radical. Diamond ${ }^{41}$ ha investigado sobre el papel que tuvieron los servicios de inteligencia americanos en la investigación, la cultura en general y en las ciencias sociales en particular. Las universidades financiadas por las fundaciones tenían fuertes vínculos con agencias como el FBI, la OSS, la CIA o el National Security Council. Los vasos comunicantes que se establecieron no fueron el resultado esporádico de la Guerra Fría, sino una relación de trabajo continuada y permanente, el maccartysmo sólo sirvió para aumentar y fortalecer esta relación. Este autor describe la cooperación de los presidentes de universidades como Harvard, Yale, Princeton o California con los organismos de espionaje y la presión que se ejerció sobre el profesorado. Él mismo fue expulsado en 1950 por el rector de la Universidad de Harvard por rehusar hablar con el FBI acerca de algunos profesores y alumnos.

Después de establecerse el National Security Council en 1947 se llevó a cabo un estudio para que se recomendase a todas las universidades que tuviesen un equipo de observadores informales que deberían colaborar con la CIA. Diamond nos presenta toda una red de profesores, administradores, alumnos e instituciones filantrópicas conectadas entre sí y con el servicio del Estado ${ }^{42}$. La Segunda Guerra Mundial creó una demanda de especialistas, entre ellos muchos intelectuales emigrados como Marcuse, Neumann, Löwenthal, etc., que trabajaron para el gobierno federal en su lucha contra el nazismo, porque conocían bien la cultura y la sociedad alemanas. Un caso muy específico fue el Russian Research Center dirigido por C. Kluckhon, en el que trabajaron también Parsons y su esposa Helen, quienes fueron objeto de interrogatorio por parte del FBI, aunque no eran sospechosos de simpatizar con la causa comunista, tal como ha mostrado posteriormente J.N. Porter ${ }^{43}$ y a pesar de su posición frente al maccartysmo ${ }^{44}$.

Esta versión de la colaboración entre fundaciones, universidades y servicios de inteligencia se une a la extendida literatura americana de que la sociedad requiere un marco de vigilancia moral e ideológica. La tutela siempre presente en la sociedad viene canalizada, en este caso, a través de la orientación y el apoyo que las fundaciones proporcionaron a las ciencias sociales. El estudio

41. Diamond, S. (1992). Compromised Campus. The collaboration of the Universities with the intelligence community, 1945-55. Nueva York: Oxford University Press.

42. El 4 de octubre de 1995 la televisión británica Channel 4 emitió un programa titulado "Hidden hands. Why did the CIA use covert funding during the 1950 s to promote the work of left-wing American abstract painters?», un reportaje con mesa redonda en la que se sentó un antiguo miembro de la CIA que a su vez era miembro del Consejo de Administración de una fundación.

43. Porter, J.N. (1996). "Talcot Parsons and the National Socialism: the case of the "ten mysterious missing letters"». Sociological Forum, vol. 11, 4, p. 603-611.

44. PARSONS, T. (1955). "MacCartysm and american social tension: a sociologist's view». Yale Rev. winter, p. 226-245. 
más reciente sobre este tema nos lo ha proporcionado F.S. Sanders con su trabajo Who paid the piper? The CIA and the cultural cold War ${ }^{45}$, en el que describe pormenorizadamente la política cultural americana y su relación con los intelectuales durante la Guerra Fría.

Unos años más tarde $\mathrm{P}$. Bourdieu ${ }^{46}$ nos dirá que aunque el dinero americano suscita sospechas y se le acusa de imperialismo, él nunca se ha fiado de explicaciones monistas que denuncian jefes de orquesta clandestinos como la CIA, que desde la sombra tiran de los hilos donde se agitan los personajes de la escena pública. Sin embargo, nadie ignora que las subvenciones de las grandes fundaciones estuvieron aliadas con ideas políticas, no en balde en los años treinta la fundación Rockefeller apoyó el proyecto presentado por el economista liberal Ch. Rist en detrimento del programa presentado por M. Mauss, socialista declarado, que estaba lejos de dar las garantías políticas indispensables. Y muchos estudiosos, entre ellos Bourdieu, piensan que después de la guerra las ciencias sociales que impulsaron estas organizaciones se redujeron en lo esencial a encuestas sociográficas totalmente desprovistas de contextualización históri$\mathrm{ca}, \mathrm{y}$ financiadas como instrumentos de control social destinados a contrarrestar los efectos de las tradiciones críticas asociadas claramente al marxismo.

En esta línea M. Pollak afirmaría unos años más tarde que «las fundaciones persiguen una política más liberal que el gobierno, siguiendo una estrategia destinada a favorecer, en los países europeos, los cambios institucionales y políticos necesarios para inmunizarlos contra las tentaciones del comunismo. La institucionalización de las ciencias sociales que tanto apoyaron tenía un papel fundamental en esta estrategia, puesto que con sus enfoques empíricos y racionales podían sustituir las tradiciones ideológicas de los europeos y los enfoques globalizantes. Además, su propensión al pragmatismo y su acción reformadora en materia de programas sociales debían contribuir, a la larga, a acercar los sistemas políticos europeos, considerados autoritarios y demasiado jerarquizados para el ideal americano» ${ }^{47}$.

Parece pues evidente que las grandes fundaciones americanas tuvieron un papel protagonista en el impulso y desarrollo de la sociología entre 1945 y 1960, que suplieron la acción del Estado y que, sea cual fuere la interpretación que se dé a este hecho, su filosofía sobre el papel de las ciencias sociales en la sociedad moderna contribuyó sustancialmente a establecer las condiciones de trabajo, el contenido y el método de esta disciplina, así como su institucionalización académica.

45. SANDERS, F.S. (1999). Who paid the piper? The CIA and the cultural cold war. Nueva York: Granta.

46. Bourdie, P. (1988). «Introduction». En MazON, B., op. cit.

47. Pollak, M. (1979). «P. Lazarsfeld fondateur d'une multinationale scientifique». Actes de la Recherche en Sciences Sociales, 25, p. 56. 